\title{
Téoros
}

Revue de recherche en tourisme

\section{Vendre Montréal en hiver : une étude de cas, 1976-1989}

\section{Pierre Labrie}

Volume 8, numéro 3, novembre 1989

Tourisme hivernal

URI : https://id.erudit.org/iderudit/1080306ar

DOI : https://doi.org/10.7202/1080306ar

Aller au sommaire du numéro

Éditeur(s)

Université du Québec à Montréal

ISSN

0712-8657 (imprimé)

1923-2705 (numérique)

Découvrir la revue

Citer cet article

Labrie, P. (1989). Vendre Montréal en hiver : une étude de cas, 1976-1989.

Téoros, 8(3), 11-13. https://doi.org/10.7202/1080306ar d'utilisation que vous pouvez consulter en ligne.

https://apropos.erudit.org/fr/usagers/politique-dutilisation/ 


\section{Vendre Montréal en hiver: Une étude de cas, 1976-1989}

\section{La loi de l'offre et de la demande \\ Premier fait d'arme: Boule de neige (Snowball)}

C'est après les Olympiques de 1976 que les intervenants touristiques du Montréal métropolitain ont commencé à se préoccuper de la mise en marché de Montréal comme destination touristique en hiver. Après Expo 1967 et avec la préparation des Olympiques, le parc hôtelier de Montréal s'était accru sensiblement; plus de 2500 nouvelles chambres d'hôtels s "etaient ajoutées à une offre touristique déjà supérieure à la demande. Le contexte économique québécois, la concurrence accrue et le développement de l'Ontario, l'accessibilité à de nouvelles destinations, autant d'éléments qui replaçaient Montréal dans un contexte concurrentiel. De plus, soulignons qu'à la même époque on était encore affecté par l'impact négatif de la crise de l'énergie de 1973 ; depuis cette date, le Canadaet, parconséquent, Montréal avaient perdu une grande partie de leur clientèle touristique traditionnelle qui avait renoncé aux voyages par automobile suite à une augmentation importante des prix de l'essence.

- Monsieur Pierre Labrie est assistant directeur aux affaires publiques, Secrétariat Genéral, Ville de Montréal. De 1980 à 1989, il a été directeur de CIDEM-Tourisme. II est également vice-president du consei d'administration du Centre d'etudes du tourisme.
C'est à l'automne 1976 que la Ville de Montréal apropose aux intervenants touristiques d'initier une opération de mise en marché de Montréal comme destination touristique en hiver. Inspirée par un concept européen, Montréal se proposait d'être la ville de séjour des visiteurs américains souhaitant skier dans les Laurentides et en Estrie. Skier le jour, découvrir les plaisirs de la ville le soir. Connue sous le nom d'opération "Snowball", cette initiative de marketing coopératif fut financée en partie par la Ville, les établissements hôteliers, les transporteurs terrestres, les transporteurs aériens. L"opération "Snowball" fut lancée en décembre 1976, la stratégie préconisée à l'époque visait à faire vendre Montréal par les grossistes en voyages spécial isés dans la revente de produits touristiques aux ËtatsUnis et en Ontario.

L'opération fut répétée en 1977-1978 et 1978-1979. Dès sa deuxième année, le programme initial qui visait à déplacer les skieurs vers les pentes de ski, matins et soirs, a beaucoup évolué. La démonstration venait d'être faite que les bâtisseurs de condominiums au pied des pentes auraient raison dans les années " 80 . Un skieur ne souhaite pas résider loin du lieu de pratique de son sport favori. Il préfère séjourner au pied des pentes. Le programme "Snowball" a donc évolué vers un concept plus urbain (et moins pentes de ski).

Il est difficile d'évaluer avec précision la rentabilité et les résultats du programme "Snowball". Peu de données chiffrées sont disponibles. Toutefois, compte tenu des efforts engagés dans la mise en marché et la diffusion du matériel de promotion, l'opération "Snowball" a certainement eu un impact en termes de visibilité, de positionnement, d'image et de diffusion d'informations sur Montréal. En trois ans, plus de $800000 \$$ ont été investis dans ce programme.

\section{Pour quelques entrechats en bottes de shi}

A l'automne 1979, la Ville de Montréal créait la Commission d'initiative et de développement économiques de Montréal et lui confiait le mandat de développer l'économie de Montréal. Ce nouveau service municipal avait pour mission de changer la façon de faire les choses, de travailler avec le secteur privé, de lui confier davantage de responsabilités et de l'impliquer systématiquement dans la réalisation de ses diverses activités. En étroite collaboration avec un Comité consultatif regroupant les intervenants du milieu touristique, diverses associations, notamment celle regroupant les directeurs de marketing des principaux établissements hôteliers de Montréal, la Ville de Montréal de concert avec ces derniers développait un nouveau concept de mise en marché de Montréal en hiver rèposant sur les critères suivants:

1. Développer et implanter un nouveau concept de vente directe aux consommateurs sans utilisation d'intermédiaires (grossistes en voyages) pour la revente. Tout en soutenant la vente aux consommateurs via des agents de voyages détaillants (protection de la commission de $10 \%$ ).

2. Développer et mettre en oeuvre un programme de publicité coopérative agressif impliquant l'ensemble des établissements participants dans la sélection des médias utilisés, dans le visuel du programme et dans le suivi de diffusion.

3. Identifier avec précision une zone géographique pour une mise en marché plus précise et correspondant au profil socio-économique et au comportement du consommateur en période hivernale (nord-est américain, Ontario et province de Québec).

4. Développer de nouvelles sources de financement impliquant entre autres la mise en commun de plus de $150000 \$$ de ressources du secteur privé (Budget 
total de la campagne environ $500000 \$$ ).

5. Encourager une politique de prix agressive, chaque établissement proposant aux consommateurs son meilleur prix possible pour un séjour à Montréal durant la période hivernale.

Cette campagne intégrée faisait appel à la publicité, à la promotion, aux envois postaux, aux relations publiques, elle reposait sur un visuel "punché" et un titre accrocheur: "Montréal the paradoxical" (version anglaise). Cette campagne a été reprise en 1981-1982. Les résultats ont été très encourageants. Plus de 7000 personnes ont acheté ces forfaits directement auprès des établissements hôteliers en 19801981 et près de 11000 en 1981-1982. C'était la première fois qu'un effort de marketing coopératif réalisé par les intervenants touristiques montréalais donnait de tels résultats. Cela amenait dès 19821983 les intervenants touristiques montréalais à se questionner sur l'importance de mettre en commun davantage de leurs ressources en termes de mise en marché. C'est à l'été 1982 que la Ville et les intervenants concernés commercialisaient un programme annuel de promotion "Montréal, it's four times better" qui comportait un volet estival et un volet hivernal. C'était la première fois depuis 1976 que les intervenants et les paliers de gouvernements mettaient en commun leurs ressources pour réaliser un effort continu de douze mois pour la mise en marché de Montréal comme destination touristique. Appuyé en 1983 et 1984 de campagnes de publicités coopératives, le programme "It's four times better" $s$ 'est ensuite poursuivi comme publication de soutien aux campagnes promotionnelles de Montréal entre 1985 et 1988.

\section{Toronto double Montréal pour son plaisir}

En 1986, les intervenants touristiques de Toronto développaient un nouveau concept de mise en marché très agressif: "Toronto double your pleasure", Cette initiative concentrée sur les mois de février et mars visait à résorber une offre abondante et une baisse importante $\mathrm{d}^{\dagger}$ achalandage. Autrement dit, baisse radicale des prix, vente de blanc au prix coûtant, valeur ajoutée sous forme de couponsrabais, question d'encourager les visiteurs à fréquenter Toronto durant la morne saison. Son succès en 1986 a donné à ce programme une grande notoriété au Canada et aux États-Unis. En 1987, les intervenants montréalais de concert avec l'Office des Congrès et du Tourisme du Grand Montréal et la Ville de Montréal convenaient que
Montréal comme destination, se devait de mettre en marché un programme similaire. Diverses raisons militaient en ce sens: nouveaux établissements höteliers à Montréal concurrence accrue en termes de tourisme au Canada, (Toronto, Québec, Ottawa) baisse anticipée du nombre de congrès et de la fréquentation de touristes d'affaires durant la période hivernale.

\section{Montréal -50 et la suite}

Tout en maintenant le programme ${ }^{44}$ It's four times better", brochure maintenant diffusće à plus de 300000 exemplaires annuellement, les intervenants montréalais ont développe, en 1987, un nouveau concept de promotion de Montréal en hiver. Ils mettaient en commun leurs ressources (O.C.T.G.M., A.H.G.M., CIDEM), développaient une stratégie intégrée et, pour la première fois, intégraient au développement d'un programme marketing des cléments de mesure de performance et de recherche qualitative. De plus, Montréal innovait en 1987-1988 en développant de nouvelles sources de financement pourcette campagne de promotion connue maintenant sous le theme de "Montréal -50 " (Budget total de 750000 \$).

En effet, Montréal innovait en sollicitant des commandites corporatives auprés de la compagnie American Express du Canada et de Via Rail. (Depuis 1988, Toronto, Québec, Ottawa et Vancouver ont tous développé une approche similaire).

Les résultats de l'opération "Montréal -50" ont été très positifs, un taux de satisfaction élevé de la part des consommateurs. Une augmentation de l'achalandage $(30 \%$ des clients du programme étaient de nouveaux clients qui n"avaient jamais visité Montréal en hiver auparavant). Elle a contribué à l'augmentation de la notoriété de Montréal. Des innovations réalisées en termes de marketing, telles 1'opération "carte postale", la publicité coopérative et la mise en commun de ressources ont été autant d'éléments qui ont donné satisfaction aux intervenants. Les donnees recueillies en termes de connaissances des clientèles, marchés, prix, activités, niveau de satisfaction ont permis de développer un produit bonifié en 1988-1989 connu sous le nom de "Montréal: Big on life this winter" ou de "Vivre Montréal en hiver" "La stratêgie marketing a également êté repensêe reposant davantage sur des efforts de publicité directe, de relations de presse, publicité coopérative, de développement de programmes d'activités tout en recherchant de nouvelles sources de financement avec un budget de $950000 \$$ pour cette campagne. Montréal se retrouvait, en 1988-1989 en concurrence avec Québecet Ottawa en plus de Toronto qui avaient maintenant développé des programmes similaires. Les résultats obtenus en 1988-1989 furent très positifs mais ne peuvent être comparés à l'année précédente car, en 1988-1989, le programme "It's four times better" a êté abandonné et ses données intégrées à l'operration de promotion hivernale; ce qui a permis d'augmenter sensiblement la diversité des forfaits achetés par les visiteurs à Montréal (plus de 50000 nuités).

\section{Une stratégie pour vendre I'hiver urbain}

Il existe des méthodes éprouvées qui, appliquées à la mise en marché de plusieurs produits, ont donné d'excellents résultats. Vendre une ville comme Montréal en hiver, cela est réalisable; cela peut donner des résultats positifs quantifiables; il s'agit de mettre en place un ensemble d'éléments et de développer une méthodologie constante. En nous référant aux données des études SECOR 1988, Laventhol \& Horwath 1988 et 1989 et aux observations recueillies au fil des ans, nous avons essayé de mettre en perspective les composantes d'une recette à succès.

\section{Élaboration des stratégies}

Quatre (4) types de stratégies doivent être envisagées pour développer de nouvelles clientèles touristiques de Montréal en hiver:

\section{A) Stratégie de produits}

Le developpenent des produits, c'est-àdire des investissements en capital pour bonifier l'offre touristique. Cela vise, entre autres, la réalisation de nouveaux équipements et attraits permanents qui contribueront à bonifier l'offre touristique (Insectarium, Biodôme au Parc Olympique, Musée d'art contemporain, Musée des Beaux-Arts au centre-ville de Montréal, etc.). Autant de projets qui, par leur qualité et leur rayonnement international contribuerontà intéresser davantage les touristes à l'offre montréalaise.

Parallèlement aux équipements, le mobilier urbain hivernal de Montréal doit être bonifié pour répondre aux impératifs de la mise en marché touristique. Dans ce sens, le projet Nordicité réalisé par la CIDEC en collaboration avec l' lssociationdes jeunes $^{\prime}$ designers a permis de créer à Montréal un type de mobilier urbain décoratif, innovateur et unique en son genre. D'ici 1992 . 
plusieurs places publiques de Montréal seront ainsi décorées. Une stratégie de développement d"une destination urbaine pour fins de tourisme hivernal prend nécessairement du temps à se réaliser. Globalement l'image de la destination se bonifiera et profitera à moyen et à long terme aux diffêrents intervenants touristiques.

\section{B) Stratégie de services}

Développer des services et activités susceptibles de bonifier le produit touristique de la destination. Au cours des trois dernières années, la Ville de Montréal a été particulièrement active à concevoir et développer des activités et des services qui contribueront à augmenter le niveau de satisfaction des clientèles touristiques visitant Montréal en hiver. Deux types d'efforts ont été particulièrement réalisés; le premier, pour bonifier la Fểte des Neiges qui se déroule à la fin du mois de janvier et lui donner une dimension régionale et nationale pour y attirer un plus grand nombre de participants de l'extérieur de la région. La Fếte des Neiges s'est donnée comme objectif de doubler la participation de touristes à des activités d'un seuil de $5 \%$ en 1991 et à $10 \%$ en 1992.

Deuxièmement, la Ville de Montréal en collaboration avec le milieu, a contribué à développer de nouveaux événements pour maximiser les fréquentations touristiques en hiver. Le premier exemple à mentionner est l'obtention du Congrès international de l'American Winter Public Works Association; congrès spécialisé dans les travaux publics d'hiver. Un autre exemple plus frappant est l'obtention, pour le mois de février 1992 du Winter Cities Showcase, événement polyvalent conçu pour la première en 1986 à Edmonton et qui se réalise à Tromsø, Norvège en 1990.

Le Winter Cities Showcase regroupe quatre événements en un: Conférence internationale des maires des villes nordiques, un Forum (Congrès) spécialisé sur les technologies de l'hiver et enfin, une foire commerciale regroupant des producteurs et des acheteurs de services associés à l'hiver. Le tout couronné par une importante animation populaire et culturelle. Le Winter Cities Showcase est un exemple concret de "produit" nouveau et innovateur créé de toute pièce pour combler le vide hivernal. Les objectifs que se fixera le Comité organiisateur en termes de nombre, taille, contribueront, par leur atteinte à augmenter la profitabilité des entreprises touristiques montréalaises durant la période hivernale.

\section{C) Stratégie "temporelle"}

Stratégie complémentaire dans le domaine des événements majeurs, il faut favoriser l'étalement des manifestations. En effet, durant la saison estivale et l'automne, Montréal est l'hôte de grandes manifestations quil se bousculent pour séduire l'attention des Montréalais et des visiteurs. Il importera, aucours des prochaines années, de favoriser un étalement de ces manifestations vers la fin d'automne et l'hiver et ce, afin d'étaler sur une plus grande période l'offre et ainsi favoriser une consommation plus soutenue de la part des clientèles montréalaises. Cela favorisera également la fréquentation de Montréal par des clientèles touristiques. Le Musée des Beaux-Arts de Montréal avec l'exposition Chagall durant 1'hiver 1988-1989 a été exemplaire à cet égard.

\section{D) Stratégie de promotion}

La mise en place des trois premières stratégies permettra de développer des stratégies pour vendre la destination. Ces stratégies reposent sur des segmentations précises dont celles de:

\section{Segmentation par but de voyage}

En premier lieu, il importe de connaitre les motifs et buts de voyages de la clientèle qui fréquente présentement Montréal. Buts tels les affaires, les congrès, l'agrément (parents et amis, voyages de groupes, weekend, etc.), les foires commerciales professionnelles et grand public. Cette segmentation complétée, pondérée en pourcentage et en nombre, nous obtenons un portrait des intentions de voyages passées et présentes de la clientèle touristique de Montréal. Un plan d'action et des objectifs de ventes pourront, à partir de cette segmentation, être développés pour chacun des segments.

\section{Segmentation géographique}

Le profil de la clientèle qui a visité Montréal durant la période hivernale 1988 était légèrement différent de celui des autres trimestres de l'année 1988. Cette analyse, confirmée par d'autres sources, permet d'identifier qu'il faut concentrer, durant la période hivernale, les efforts de marketing à un rayon d'intervention plus restreint. Ainsi, les efforts de mise en marché de Montréal comme destination en période hivernale porteront davantage sur le Québec, l'est de l'Ontario (Toronto et Ottawa) et le nord-est américain incluant les régions de New York et Boston.
La stratégie de promotion devra être bien synchronisée avec le processus décisionnel des consommateurs (bon moment), récurrente pour établir sa notoriété et capitaliser sur ses acquis, faire appel aux facteurs les plus susceptibles de générer [et de l'intérêtt] des ventes (les prix!) [et un rapport qualité/prix].

\section{Conclusion}

Vendre Montréal en hiver est un défi que l'on doit relever constamment. Il ne faut pas perdre de vue que Montréal comme destination hivernale est en concurrence avec les promoteurs de vacances dans le sud. Il faut être réaliste car une destination touristique quelle qu'elle soit aura toujours une haute saison et une basse saison. Ville d'été, l'hiver à Montréal fera toujours partie, sauf exception, de la basse saison. II s"agit donc de maximiser le potentiel urbain hivernal en misant sur ces points forts et ces spécificités. $\boldsymbol{f}$ 